\title{
The effect of stromal hydration on surgical outcomes for cataract patients who received a hydrogel ocular bandage
}

This article was published in the following Dove Press journal:

Clinical Ophthalmology

I8 March 201 I

Number of times this article has been viewed

\author{
Thomas R Walters \\ Texan Eye, Austin, TX, USA
}

Correspondence: Thomas R Walters Texan Eye, 5717 Balcones Drive, Austin, TX 7873I, USA

Tel + I 5123277000

Fax + I 5124544928

Email twalters@austin.rr.com
Purpose: The purpose of this study was to examine the effect of stromal hydration on surgical outcomes for patients who received the new hydrogel ocular bandage (ReSure ${ }^{\mathrm{TM}}$ Adherent Ocular Bandage, Ocular Therapeutix, Inc, Bedford, MA, USA) following routine cataract surgery.

Methods: This post-hoc, single-masked study was conducted with 310 patients who were scheduled to undergo unilateral clear corneal cataract surgery with phacoemulsification and intraocular lens implantation. Incisions were closed with stromal hydration ( 270 patients) or without stromal hydration ( 40 patients) based on physician standard of care. All patients received the hydrogel bandage at the conclusion of the procedure. Ocular assessments of stromal edema, flare, corneal staining, anterior chamber cells, best-corrected visual acuity, and intraocular pressure were made 24 hours after surgery.

Results: Significantly more patients experienced stromal edema in the group with stromal hydration $(26.3 \%$ versus $10.0 \%$, respectively; $P=0.028)$. A higher percentage of patients experienced corneal staining when stromal hydration was performed $(20.4 \%$ versus $2.5 \%$; $P=0.004$ ). The mean BCVA (best-corrected visual acuity) also was significantly different between the groups (logMAR of 0.164 with stromal hydration versus 0.095 without hydration; $P=0.007)$. No significant differences were observed between the study groups in terms of flare, anterior chamber cells, or intraocular pressure.

Conclusion: Cataract surgery without stromal hydration provided better surgical outcomes than the traditional hydration procedure when used in conjunction with a new hydrogel bandage.

Keywords: cataract surgery, hydrogel bandage, phacoemulsification, stromal hydration

\section{Introduction}

Cataract surgery has become one of the most frequent surgeries performed in the US. ${ }^{1}$ An American Society of Cataract and Refractive Surgery survey reported that over 3.1 million cataract surgeries were performed in $2008 .^{2}$ The clear corneal incision (CCI) technique was introduced by Fine in the early $1990 \mathrm{~s} .{ }^{3-5}$ This technique is widely preferred today because few or no sutures are required.

Stromal hydration is often performed in conjunction with the CCI in an attempt to close the wound, ${ }^{6}$ even though studies have shown that the integrity of the CCI may be compromised immediately after surgery ${ }^{7,8}$ with gaping of the wound in the early post-surgical period. Therefore, even uncomplicated CCIs may allow the ingress of ocular surface bacteria. ${ }^{9}$

As surgical techniques continue to advance, alternatives to the standard procedures may provide benefits in terms of improved outcomes for the patient. Extra care taken at the time of surgery to ensure that the incision is properly sealed should further minimize 
the risks of later complications. One means of achieving this has been by performing stromal hydration of the wound, but now other methods are becoming available.

A new hydrogel bandage has recently been developed. ${ }^{10-13}$ This device is made from polyethylene glycol, trilysine, buffering salts, and water. The bandage is applied to the incision as a liquid that then forms a gel within about 30 seconds. The bandage is formulated with a blue colorant to enhance visualization. This color diffuses out of the device within a few hours of application. Although this hydrogel technology platform is being used successfully in other areas of medicine, this particular device was specifically developed to protect ophthalmic incisions.

The purpose of the current post-hoc study was to determine whether stromal hydration contributes to persistent corneal edema and whether it has any effect on best-corrected visual acuity (BCVA) in the immediate postoperative period (24 hours).

\section{Methods}

This was a post-hoc analysis performed on a subset of data from a controlled, prospective, randomized, parallel-group multicenter study $(\mathrm{n}=420)$ comparing the ReSure Adherent Ocular Bandage (Ocular Therapeutix, Inc) with the Oasis 24-hour Soft Shield ${ }^{\circledR}$ Collagen Corneal Shield (Oasis Medical, Glendora, CA, USA) for protection and relief of pain and discomfort following cataract surgery. ${ }^{12}$ The current analysis included all the hydrogel bandage patients $(n=310)$ from the original study (except for six patients who received sutures), which comprised 40 patients who did not undergo stromal hydration during surgery and 270 who did receive hydration. The decision not to use stromal hydration was based on surgeon's preference rather than on the complexity of each case.

Detailed methods of the prospective randomized clinical trial are described by Dell et al ${ }^{12}$ Briefly, the study included adults who were scheduled for unilateral clear corneal cataract surgery with phacoemulsification and implantation of a posterior chamber intraocular lens through a $\leq 3.5 \mathrm{~mm}$ incision. The surgery was performed using a short-acting (duration of about 10-12 minutes) topical anesthetic agent (lidocaine, proparacaine, or tetracaine). If required, topical anesthesia was supplemented with intracameral anesthesia also using a short-acting agent (eg, lidocaine).

Exclusion criteria included an active ocular infection, a history of or active intraocular inflammation, glaucoma or ocular hypertension ( $\geq 25 \mathrm{mmHg}$ ), macular degeneration, previous ocular surgery or ocular abnormality, planned combination surgery, and BCVA of the fellow eye of less than 20/40. In addition, patients were considered screening failures and were not eligible to be randomized into the study if the immediate postoperative eye was observed to have a wound leakage (positive Seidel test), with or without stromal hydration and/or suture placement. Informed consent was obtained from all patients. The RCRC Independent Review Board (Austin, TX) approved this study.

At the conclusion of the cataract surgery patients received stromal hydration or not, based on standard physician practice. All subjects (100\%) had a negative Seidel test demonstrating no leakage. Upon confirmation that the main incision was not leaking, the hydrogel ocular bandage was reconstituted and applied to the incision site. Figure 1 shows the device on the corneal surface. Patients in both groups received fourth-generation fluoroquinolone antibiotic and corticosteroid eye drops in the operative eye following application of the hydrogel bandage. Topical and/or oral nonsteroidal anti-inflammatory drugs (NSAIDs) or other oral pain medications were prohibited until after the 24-hour pain assessment. Following the 24-hour pain assessment, the postoperative medication regimen used was as the standard of care for the institution, and the same regimen was applied to both treatment groups. The majority of patients received antibiotics, steroids, and NSAIDs. Patients were then discharged with the eye uncovered.

The study patients underwent a complete preoperative eye examination including BCVA (via Early Treatment Diabetic Retinopathy Study), intraocular pressure (IOP) measurement,

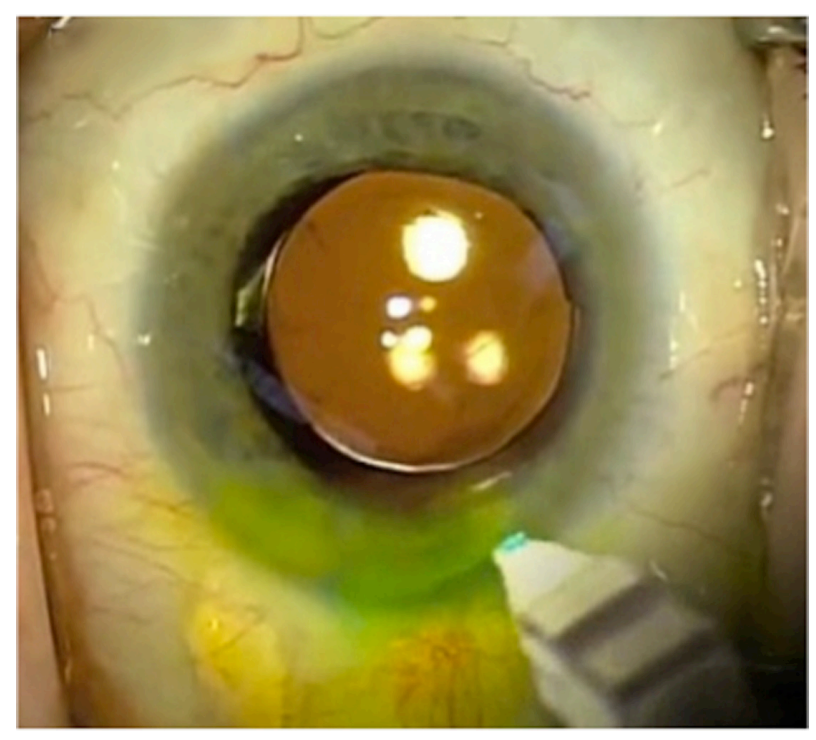

Figure I Representative photograph of the hydrogel ocular bandage being applied on the ocular surface following cataract surgery. Note that the yellow coloring is from the fluorescein staining of a Seidel test. Photo courtesy of Dr Samuel Masket. 
biomicroscopy, and dilated ophthalmoscopy. Postoperative evaluations at 24 hours were the subject of this analysis.

\section{Statistical analyses}

All statistical tests were two-sided, and the significance level was set at $5 \%$. The two sample $t$-test was used to test for differences in means between treatment groups for continuous variables. Fisher's exact test was used to test for differences in proportions between groups for categorical variables. The analyses were conducted in a post-hoc manner, and there was no multiplicity adjustment. All statistical analyses were performed using $\mathrm{SAS}^{\circledR}$ Version 9.1 (SAS Institute, Cary, NC, USA).

\section{Results}

Summary statistics of demographics and surgical parameters are provided in Tables 1 and 2, respectively. The two groups were similar in terms of age, gender, race, and incision width. The mean duration of surgery was 13.5 minutes and 12.2 minutes with and without stromal hydration, respectively. A temporal incision was used exclusively in the study group without stromal hydration. Most of the incisions were multiplane (39 of 40) for the group without stromal hydration.

A significantly higher percentage of patients with stromal hydration experienced stromal edema at 24 hours $(26.3 \%$ versus $10.0 \% ; P=0.028$ ) (Table 3 ; Figure 2). Results from the 24-hour ocular assessments are provided in Table 3.

Corneal staining was observed in $20.4 \%$ of the patients who underwent stromal hydration compared with only $2.5 \%$ in the group without stromal hydration. This difference was statistically significant ( $P=0.004$ ) (Table 4; Figure 3). BCVA was also significantly better in the group without stromal hydration $(P=0.007)$ (Table 3 ; Figure 4$)$.

\section{Safety}

The safety population consisted of all 310 patients included in the post-hoc analysis. Sixty-seven patients $(21.6 \%)$ reported at least one adverse event over the study follow-up duration of 30 days. An increase in IOP to $\geq 30 \mathrm{mmHg}$ or $10 \mathrm{mmHg}$ over baseline was observed for 35 of 310 (11.3\%) patients; 32 of $270(11.9 \%)$ in the stromal hydration group versus 3 of $40(7.5 \%)$ in the group without stromal hydration. A worsening in BCVA of $>2$ lines ( $>10$ letters) was seen for 25 of $310(8.1 \%)$ patients; 23 of $270(8.5 \%)$ in the stromal hydration group versus 2 of $40(5.0 \%)$ in the group without stromal hydration. Other adverse events occurred with a frequency of $<1 \%$. There were no serious or unanticipated adverse events. The adverse events experienced are consistent with typical post-cataract outcomes.

\section{Discussion}

Stromal hydration is a technique that has been used following phacoemulsification since the early 1990s and is still widely used today. ${ }^{5,6}$ There have been ongoing discussions in the literature around the merits of this technique. . $^{67,14-19}$ In a study involving 80 eyes that received $2.2 \mathrm{~mm}$ incisions, Vasavada and colleagues reported a reduced ingress of trypan blue that had been instilled on the ocular surface. ${ }^{6}$ Fine and others suggest that the stromal swelling that results from the hydration technique may persist for at least 24 hours. ${ }^{16,19}$ Due to the fact that hydration can improve wound closure,

Table I Patient demographics

\begin{tabular}{|c|c|c|c|c|}
\hline Parameter & Statistic & $\begin{array}{l}\text { With stromal hydration } \\
(n=270)\end{array}$ & $\begin{array}{l}\text { Without stromal } \\
\text { hydration }(n=40)\end{array}$ & $P$-value ${ }^{a}$ \\
\hline \multirow[t]{4}{*}{ Age (years) } & Mean & 69.2 & 65.8 & 0.055 \\
\hline & Median & 69.5 & 66.0 & \\
\hline & SD & 10.08 & 12.10 & \\
\hline & Minimum - Maximum & $32-93$ & $20-89$ & \\
\hline Sex & & n (\%) & n (\%) & 1.000 \\
\hline Female & & 134 (49.6) & $20(50.0)$ & \\
\hline Male & & $136(50.4)$ & $20(50.0)$ & \\
\hline Race & & n (\%) & n (\%) & 0.098 \\
\hline Caucasian & & $252(93.3)$ & $36(90.0)$ & \\
\hline Native American & & $0(0.0)$ & $0(0.0)$ & \\
\hline African-American & & $7(2.6)$ & $0(0.0)$ & \\
\hline Hispanic & & $5(1.9)$ & $4(10.0)$ & \\
\hline Asian-American & & $4(1.5)$ & $0(0.0)$ & \\
\hline Other & & $2(0.7)$ & $0(0.0)$ & \\
\hline
\end{tabular}

Note: a ${ }^{P}$-value is based on the two-sample $t$-test for continuous variables or Fisher's exact test for categorical variables. Abbreviation: SD, standard deviation. 
Table 2 Surgical parameters

\begin{tabular}{llll}
\hline Parameter & Statistic & $\begin{array}{l}\text { With stromal hydration } \\
(\mathbf{n = 2 7 0 )}\end{array}$ & $\begin{array}{l}\text { Without stromal hydration } \\
(\mathbf{n}=\mathbf{4 0})\end{array}$ \\
\hline Duration of surgery $(\mathrm{min})$ & Mean & 13.5 & 12.2 \\
& Median & 12.0 & 11.0 \\
& SD & 5.25 & 3.25 \\
& Minimum - Maximum & $5-38$ & $8-24$ \\
Incision width $(\mathrm{mm})$ & $\mathrm{n}$ & 270 & 40 \\
& Mean & 2.68 & 2.69 \\
& Median & 2.70 & 2.70 \\
& SD & 0.292 & 0.078 \\
Incision type & Minimum - Maximum & $2.1-3.4$ & $2.4-2.8$ \\
Single piece & & $\mathrm{n}(\%)$ & $\mathrm{n}(\%)$ \\
Multiplane & $144(53.3)$ & $1(2.5)$ \\
Incision location & $126(46.7)$ & $39(97.5)$ \\
Temporal & $\mathrm{n}(\%)$ & $\mathrm{n}(\%)$ \\
Supra temporal & $214(79.3)$ & $40(100.0)$ \\
Nasally & & $39(14.4)$ & $0(0.0)$ \\
Supra nasally & $2(0.7)$ & $0(0.0)$ \\
Superior & $7(2.6)$ & $0(0.0)$ \\
\hline
\end{tabular}

Abbreviation: SD, standard deviation.

this procedure has been recommended as a routine step after phacoemulsification. ${ }^{6}$

Some caveats to stromal hydration have been pointed out in recent studies. Calladine and Packard examined 34 adult eyes using the Carl Zeiss Visante anterior segment optical coherence tomography (OCT) imaging system within one hour of cataract surgery. ${ }^{7}$ The investigators performed stromal hydration on the main incision only if they suspected leakage.

The authors reported that stromal hydration tended to increase corneal thickness around the incision, which also increased the incision length significantly. They went on to suggest that additional stromal hydration in the main incision might not routinely be necessary at the end of the procedure.

Behrens and colleagues used $\mathrm{OCT}$ to examine $\mathrm{CCI}$ incisions 24 hours following uneventful phacoemulsification surgery. ${ }^{8}$ The authors found that small-incision clear cornea wounds that have received stromal hydration can gape in the immediate postoperative period. Descemet's membrane detachment was

Table 3 Ocular assessments 24 hours after cataract surgery

\begin{tabular}{llll}
\hline Parameter & Statistic & $\begin{array}{l}\text { With stromal hydration } \\
(\mathbf{n}=\mathbf{2 7 0})\end{array}$ & $\begin{array}{l}\text { Without stromal hydration } \\
(\mathbf{n}=\mathbf{4 0})\end{array}$ \\
\hline Stromal edema & & $\mathrm{n}(\%)$ & $\mathrm{n}(\%)$ \\
Yes & & $71(26.3)$ & $4(10.0)$ \\
No & & $199(73.7)$ & $36(90.0)$ \\
Flare & & $\mathrm{n}(\%)$ & $\mathrm{n}(\%)$ \\
Yes & & $114(42.2)$ & $16(40.0)$ \\
No & & $156(57.8)$ & $24(60.0)$ \\
Anterior chamber cells & & $\mathrm{n}(\%)$ & $\mathrm{n}(\%)$ \\
Yes & & $225(83.3)$ & $38(95.0)$ \\
No & & $45(16.7)$ & $2(5.0)$ \\
BCVA & $\mathrm{n}$ & 267 & 40 \\
& Mean & 0.164 & 0.095 \\
& Median & 0.120 & 0.040 \\
& SD & 0.2128 & 0.1358 \\
IOP & Minimum - Maximum & $-0.18-1.08$ & $-0.08-0.52$ \\
& $\mathrm{n}$ & 270 & 40 \\
& Mean & 19.3 & 17.8 \\
& Median & 18.0 & 17.0 \\
& SD & 6.75 & 4.81 \\
\hline
\end{tabular}

Note: ${ }^{a} P$-value is based on the two-sample $t$-test for continuous variables or Fisher's exact test for categorical variables.

Abbreviations: SD, standard deviation; BCVA, best corrected visual acuity; IOP, intraocular pressure. 


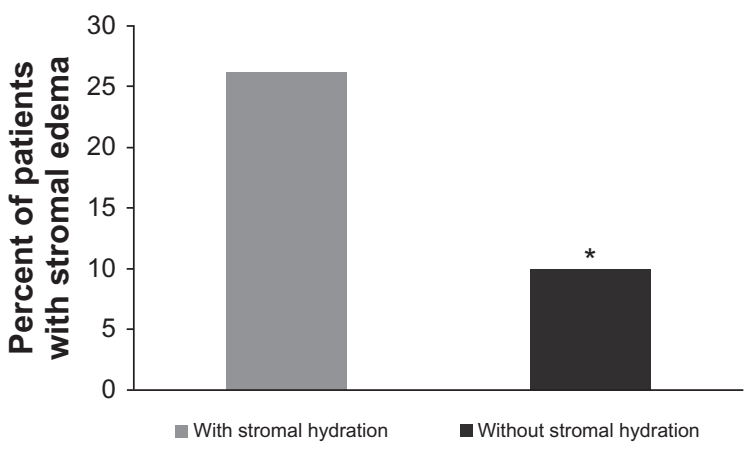

Figure 2 Percentage of patients with stromal edema 24 hours after cataract surgery. The difference between the study groups was statistically significant $(* P=0.028)$. All patients received a hydrogel bandage after surgery.

observed using the OCT and was suggested to be more common than traditionally observed using slit-lamp microscopy. Stromal hydration may be one cause of Descemet's membrane detachment during surgery. ${ }^{7,15}$ These results raise into question whether stromal hydration is optimal for sealing the wound in all CCI cases.

A recently published study evaluated whether the new hydrogel bandage could create a watertight seal in a clear corneal incision. ${ }^{11}$ Twenty-four CCIs were made in eye bank corneas that were mounted to an artificial anterior chamber. Twelve of the wounds were sealed with the hydrogel bandage while the other twelve were not. A Seidel test was performed to assess leakage of the wounds. If the test was positive, stromal hydration of the incision was performed. Three eyes in each group required stromal hydration. The mean measured IOP was $36 \mathrm{mmHg}$ in the control group and $34 \mathrm{mmHg}$ in the bandage-treated group. Ingress of India ink was observed in $75 \%(9 / 12)$ of the control group and none $(0 / 12)$ of the hydrogel bandage group. In addition, fluid leakage out of the wound was seen in $92 \%$ (11/12) of the control eye and none $(0 / 12)$ of the bandage-treated eyes. Of particular note, the hydrogel bandage created a watertight seal in all of these clear corneal incisions whether stromal hydration was performed or not.

Recent studies have evaluated the new hydrogel bandage for its ability to seal ocular incisions. One laboratory study was conducted to evaluate the ability of the hydrogel bandage to seal sutureless pars plana vitrectomy sclerotomies performed on human globes procured from an eye bank. ${ }^{13}$ The incisions received either a hydrogel device, a suture, or neither and were then evaluated for the ingress of India ink. The bandage prevented the entry of ink particles in all covered incisions (11 of 11). One sutured eye ( 1 of 5 ) and four unsutured eyes (4 of 5) permitted the ingress of ink through the incision.

The recent clinical trial referenced previously by Dell and colleagues evaluated the safety and efficacy of the hydrogel bandage. ${ }^{12}$ This prospective randomized controlled clinical study of over 400 patients showed that the device was well tolerated when placed over a cataract incision. The bandage provided coverage over the incision at 24 hours after surgery in significantly more patients $(78.6 \%)$ compared with a corneal shield $(26.5 \% ; P<0.001)$.

Calladine et al used optical coherence tomography to assess the hydrogel ocular bandage following clear corneal incisions during in cataract surgery. ${ }^{20}$ Patients were randomized to either the hydrogel bandage group $(n=22)$ or the control group $(n=23)$. Stromal hydration was performed on the side-port incisions, but the main incision was not hydrated. The incisons were examined at 2 hours, at 24 hours, and at 7 days postoperatively using OCT imaging, a slitlamp fluorescein 2\% Seidel test, and Goldman applanation tonometry. In the hydrogel bandage group, all incisions were Seidel negative. One main incision was Seidel positive in the control group. In the bandage group, architectural features of the incision were covered with a smooth layer of ocular bandage. Conversely in the control group these features were exposed to the ocular surface. Immediately after surgery, IOP values were significantly lower in the control group $(13.4 \pm 5.28 \mathrm{mmHg})$ than the bandage group $(19.4 \pm 5.94 \mathrm{mmHg} ; P<0.001)$. One eye in the control group had an IOP of $5 \mathrm{mmHg}$ immediately post surgery. At days 1 and 7, there were no significant differences in IOP between the two groups. The investigators concluded that the hydrogel bandage protected the clear corneal incisions, helped to maintain a desirable postoperative IOP, and cleared rapidly from reepithelialized areas.

Results from this post-hoc analysis showed a more favorable surgical profile for patients who received the hydrogel bandage and did not undergo stromal hydration. Fewer patients experienced stromal edema and corneal staining with this surgical regimen (Tables 3 and 4; Figures 2 and 3).

Table 4 Corneal staining/erosion results for patients who received a hydrogel bandage following routine cataract surgery

\begin{tabular}{|c|c|c|c|c|}
\hline Corneal staining/erosion & Statistic & $\begin{array}{l}\text { With stromal hydration } \\
(n=270)\end{array}$ & $\begin{array}{l}\text { Without stromal hydration } \\
(n=40)\end{array}$ & $P$-value \\
\hline Yes & $\mathrm{n}(\%)$ & $55(20.4)$ & I (2.5) & 0.004 \\
\hline No & n (\%) & $215(79.6)$ & $39(97.5)$ & \\
\hline
\end{tabular}




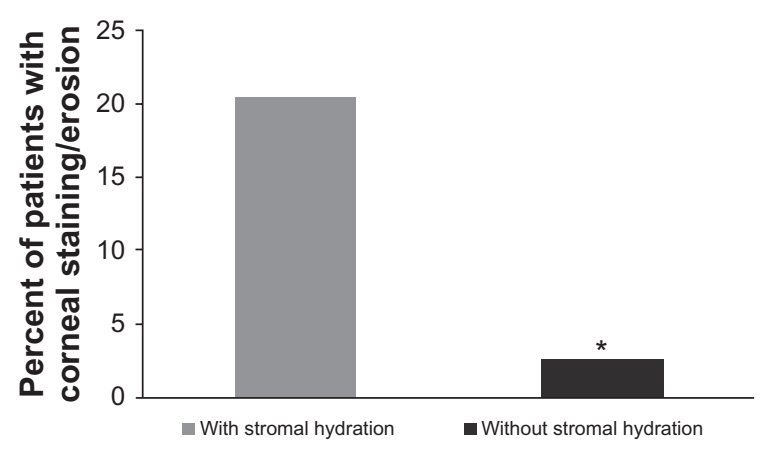

Figure 3 Percentage of patients with corneal staining/erosion 24 hours after cataract surgery. The difference between the two groups was statistically significant $(* P=0.004)$. All patients received a hydrogel bandage after surgery.

Visual acuity was also significantly better (Table 3; Figure 4) for patients who did not undergo stromal hydration. The improvement in vision 1 day postoperatively is a significant factor for patient satisfaction, particularly for self-pay patients receiving multifocal IOLs. Although not statistically significant, a lower percentage of these patients had anterior chamber cells, and their mean IOP was slightly $(1.5 \mathrm{mmHg})$ lower (Table 3). There was also a tendency toward a shorter duration of surgery ( $\sim 1$ minute) in the group of patients that did not undergo stromal hydration, as there were fewer steps (ie, less manipulation) in the overall procedure (Table 2).

One surgeon (TW) in the study did not routinely use stromal hydration. The rationale for this practice is as follows: When a surgeon chooses to hydrate, the wound may be sealed initially. However, due to the endothelial pumping mechanism, the tissues lose this hydration over time and the wound returns to its initial hydration state. This can be an issue if the initial wound architecture was not optimized and permits leaking. In short, the surgeon does not always know if the wound is structurally sound if it is hydrated. By ensuring

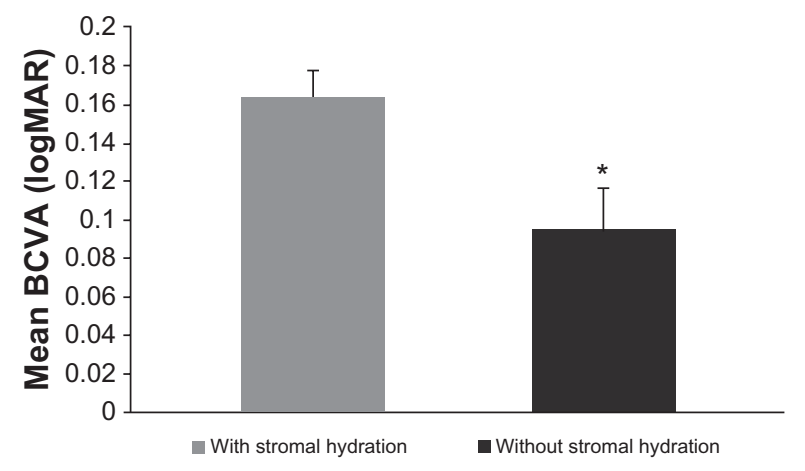

Figure 4 Mean logMAR best-corrected visual acuity (BCVA) 24 hours after cataract surgery. The difference between the groups with stromal hydration and without stromal hydration was statistically significant $(* P=0.007)$. All patients received a hydrogel ocular bandage after surgery. structural integrity of the incision at the outset and without hydration, the surgeon can be reasonably certain that the wound will not leak later due to the loss of the hydration.

As is the case with any clinical study, the current trial has some limitations. Most of the patients without stromal hydration were from one physician only. Therefore, it is not possible to separate treatment effect from surgeon effect. There was randomization in the prospective pivotal trial (NCT00774228), though no randomization between the treatments of interest was possible in the current study due to the retrospective nature of the analysis. There was a difference between the groups with regard to the type of incision (single versus multiplane). These factors should be kept in mind when making conclusions based on the findings from this study. Additional, prospective, randomized trials (possibly involving OCT) are warranted in order to explore the effects of stromal hydration, in more depth.

\section{Summary}

Stromal hydration is standard of care today, and thus often used in an attempt to oppose the two faces of an incision. However, recent evidence from the literature suggests that stromal hydration may not be optimal for favorable outcomes in all cases, ${ }^{7,8}$ due to induced wound gape allowing bacterial invasion, variability of wound architecture and integrity during the postoperative period, tissue trauma, increased surgical time, and persistent corneal edema with loss of BCVA. With the movement toward "touchless" and minimally invasive surgery, leaving the wound without stromal hydration may be favorable as this practice alters the tissues and wound as little as possible. The result is less deformation, a shorter surgical procedure, and a more rapid recovery. From the results of this analysis, favorable surgical outcomes may be expected by omitting stromal hydration, as less edema and better postoperative vision were observed in patients who did not undergo stromal hydration. In addition, to ensure integrity of the wound in a nondeformed state, the new hydrogel bandage may be optimal.

\section{Acknowledgments}

The Ocular Bandage Study Group Investigators:

Robert Cionni, MD (Eye Institute of Utah, Salt Lake City, UT); Y Ralph Chu, MD (Chu Vision Institute, Bloomington, MN); Alan S Crandall, MD (Eye Institute of Utah, Salt Lake City, UT); Steven J Dell, MD (Texan Eye, Austin, TX); Uday Devgan, MD (Maloney Vision Institute, Los Angeles, CA); John Doane, MD (Discover Vision Surgery and Laser Center, Leawood, KS); Michael Endl, MD (Fichte, 
Endl and Elmer Eyecare, Amherst, NY); William Fishkind, MD (Northwest Eye Specialists, Tucson, AZ and Clinical Professor of Ophthalmology, University of Utah, Salt Lake City, UT); William J Flynn, MD (R and R Eye Research, San Antonio, TX); Nicole Fram, MD (Advanced Vision Care, Los Angeles, CA); David R Hardten, MD (Minnesota Eye Consultants, Minneapolis, MN); John A Hovanesian, MD (Harvard Eye Associates, Laguna Hills, CA); Stephen Lane, MD (Associated Eye Care, Stillwater, MN); Jeffrey Levenson, MD (Levenson Eye Associates, Jacksonville, FL); Richard Lewis, MD (Grutzmacher and Lewis, Inc., Sacramento, CA); Samuel Masket, MD (Advanced Vision Care, Los Angeles, CA); Mark Packer, MD, FACS (Clinical Associate Professor, Oregon Health and Science University, Portland, OR and Drs Fine, Hoffman and Packer, Eugene, OR); Michael B Raizman, MD (Ophthalmic Consultants of Boston, Boston, MA); Michael Snyder, MD (Cincinnati Eye Institute, Cincinnati, OH); Thomas R Walters, MD (Texan Eye, Austin, TX).

The author would like to acknowledge Julie Crider, PhD, (Collaborative Medical Writing, LLC) for medical writing contributions and Rong Lin, MD, MPH (Biostatistical Consulting, Inc) for biostatistics.

\section{Financial disclosures}

This study was supported by Ocular Therapeutix (Bedford, MA). Dr Walters was an investigator on this study.

\section{Note}

This study has been registered with www.clinicaltrials.gov as NCT00774228.

\section{References}

1. Cataracts. 2010. http://www.allaboutvision.com/conditions/cataracts. htm. Accessed July 5, 2010.

2. Leaming D. Highlights of the 2008 ASCRS member practice style survey. San Francisco, CA: American Society of Cataract and Refractive Surgery; 2009.
3. Fine IH. Self-sealing corneal tunnel incision for small-incision cataract surgery. Ocular Surg News. 1992:38-39.

4. AAO. Cataract in the Adult Eye. Preferred Practice Patterns. 2006 : $16-26$.

5. Fine IH. Corneal tunnel incision with a temporal approach. In: Fine IH, Fichman RA, Grabow HB, editors. Clear-Corneal Cataract Surgery and Topical Anesthesia. Thorofare, NJ: Slack; 1993:25.

6. Vasavada AR, Praveen MR, Pandita D, et al. Effect of stromal hydration of clear corneal incisions: quantifying ingress of trypan blue into the anterior chamber after phacoemulsification. $J$ Cataract Refract Surg. 2007;33:623-627.

7. Calladine D, Packard R. Clear corneal incision architecture in the immediate postoperative period evaluated using optical coherence tomography. J Cataract Refract Surg. 2007;33:1429-1435.

8. Behrens A, Stark WJ, Pratzer KA, McDonnell PJ. Dynamics of small-incision clear cornea wounds after phacoemulsification surgery using optical coherence tomography in the early postoperative period. $J$ Refract Surg. 2008;24:46-49.

9. McDonnell PJ, Taban M, Sarayba M, et al. Dynamic morphology of clear corneal cataract incisions. Ophthalmology. 2003;110:2342-2348.

10. Hovanesian JA, Karageozian VH. Watertight cataract incision closure using fibrin tissue adhesive. J Cataract Refract Surg. 2007;33: 1461-1463.

11. Hovanesian JA. Cataract wound closure with a polymerizing liquid hydrogel ocular bandage. J Cataract Refract Surg. 2009;35:912-916.

12. Dell SJ, Hovanesian JA, Raizman MB, et al. Randomized comparison of post-operative use of the ReSure ${ }^{\mathrm{TM}}$ Ocular Bandage and the Collagen Corneal Shield for wound protection and patient tolerability following cataract surgery. J Cataract Refract Surg. 2011;37(1):113-121.

13. Singh A, Hosseini M, Hariprasad SM. Polyethylene glycol hydrogel polymer sealant for closure of sutureless sclerotomies: a histologic study. Am J Ophthalmol. 2010;150:346-351.

14. Vasavada AR, Mamidipudi PR, Gajjar D, Vasavada V, Raj S. Benefits of stromal hydration. J Cataract Refract Surg. 2010;36:530.

15. Calladine D, Tanner V. Optical coherence tomography of the effects of stromal hydration on clear corneal incision architecture. $J$ Cataract Refract Surg. 2009;35:1367-1371.

16. Fine IH, Hoffman RS, Packer M. Profile of clear corneal cataract incisions demonstrated by ocular coherence tomography. J Cataract Refract Surg. 2007;33:94-97.

17. Hu YJ, Hou P, Chen WQ. Factors affecting stromal hydration of clear corneal incision architecture. J Cataract Refract Surg. 2010;36:528; author reply 529.

18. Sharma V, Sharma S, Paul S, Gupta B. Effect of stromal hydration on clear corneal incisions. J Cataract Refract Surg. 2007;33:1673-1674; author reply 1674 .

19. Vasavada AR, Dholakia SA. Corneal hydration intra-operatively during phacoemulsification. Indian J Ophthalmol. 2005;53:249-253.

20. Calladine D, Ward M, Packard R. Adherent ocular bandage for clear corneal incisions used in cataract surgery. J Cataract Refract Surg. 2010;36:1839-1348.
Clinical Ophthalmology

\section{Publish your work in this journal}

Clinical Ophthalmology is an international, peer-reviewed journal covering all subspecialties within ophthalmology. Key topics include: Optometry; Visual science; Pharmacology and drug therapy in eye diseases; Basic Sciences; Primary and Secondary eye care; Patient Safety and Quality of Care Improvements. This journal is indexed on Submit your manuscript here: http://www.dovepress.com/clinical-ophthalmology-journal

\section{Dovepress}

PubMed Central and CAS, and is the official journal of The Society of Clinical Ophthalmology (SCO). The manuscript management system is completely online and includes a very quick and fair peer-review system, which is all easy to use. Visit http://www.dovepress.com/ testimonials.php to read real quotes from published authors. 